\title{
Systematic study of near-infrared intersubband absorption of polar and semipolar GaN/AIN quantum well
}

\author{
Houssaine Machhadani, M Beeler, S Sakr, E Warde, Y Kotsar, M Tchernycheva, M P.
}

Chauvat, P Ruterana, G Nataf, Ph De Mierry, E Monroy and F H. Julien

\section{Linköping University Post Print}

\section{Tweet}

N.B.: When citing this work, cite the original article.

Original Publication:

Houssaine Machhadani, M Beeler, S Sakr, E Warde, Y Kotsar, M Tchernycheva, M P. Chauvat, P Ruterana, G Nataf, Ph De Mierry, E Monroy and F H. Julien, Systematic study of near-infrared intersubband absorption of polar and semipolar GaN/AlN quantum well, 2013, Journal of Applied Physics, (113), 14.

http://dx.doi.org/10.1063/1.4801528

Copyright: American Institute of Physics (AIP) http://www.aip.org/

Postprint available at: Linköping University Electronic Press http://urn.kb.se/resolve?urn=urn:nbn:se:liu:diva-93977 


\title{
Systematic study of near-infrared intersubband absorption of polar and semipolar GaN/AIN quantum wells
}

\author{
H. Machhadani, ${ }^{1,2, a)}$ M. Beeler, ${ }^{3}$ S. Sakr, ${ }^{1}$ E. Warde, ${ }^{1}$ Y. Kotsar, ${ }^{3}$ M. Tchernycheva, ${ }^{1}$ \\ M. P. Chauvat, ${ }^{4}$ P. Ruterana, ${ }^{4}$ G. Nataf,${ }^{5}$ Ph. De Mierry, ${ }^{5}$ E. Monroy, ${ }^{3}$ and F. H. Julien ${ }^{1}$ \\ ${ }_{1}^{1}$ Institut d' Electronique Fondamentale, Université Paris-Sud, UMR 8622 CNRS, 91405 Orsay, France \\ ${ }^{2}$ Semiconductor Materials, Department of Physics, Chemistry, and Biology (IFM), Linköping University, \\ S-58183 Linköping, Sweden \\ ${ }^{3}$ CEA-CNRS Group Nanophysique et Semiconducteurs, INAC/SP2M/NPSC, CEA-Grenoble, \\ 17 rue des Martyrs, 38054 Grenoble Cedex 9, France \\ ${ }^{4}$ CIMAP, UMR 6252, CNRS-ENSICAEN-CEA-UCBN, 6 Bd Maréchal Juin, 14050 Caen, France \\ ${ }^{5}$ CRHEA, UPR 10, 1 rue Bernard Grégory, Sophia Antipolis, 06560 Valbonne, France
}

(Received 23 January 2013; accepted 25 March 2013; published online 12 April 2013)

\begin{abstract}
We report on the observation of intersubband absorption in GaN/AlN quantum well superlattices grown on (11) 2 )-oriented GaN. The absorption is tuned in the $1.5-4.5 \mu \mathrm{m}$ wavelength range by adjusting the well thickness. The semipolar samples are compared with polar samples with identical well thickness grown during the same run. The intersubband absorption of semipolar samples shows a significant red shift with respect to the polar ones due to the reduction of the internal electric field in the quantum wells. The experimental results are compared with simulations and confirm the reduction of the polarization discontinuity along the growth axis in the semipolar case. The absorption spectral shape depends on the sample growth direction: for polar quantum wells the intersubband spectrum is a sum of Lorentzian resonances, whereas a Gaussian shape is observed in the semipolar case. This dissimilarity is explained by different carrier localization in these two cases. (C) 2013 AIP Publishing LLC [http://dx.doi.org/10.1063/1.4801528]
\end{abstract}

\section{INTRODUCTION}

Since the pioneering work of West and Eglash in 1984, ${ }^{1}$ intersubband (ISB) transitions in semiconductor quantum wells (QWs), i.e., the transitions between the confined levels within the same band, have attracted great attention. This interest has been motivated by the development of a new kind of optoelectronic devices based on ISB transitions such as quantum cascade lasers (QCLs) $)^{2,3}$ and quantum well infrared photodetectors (QWIPs). ${ }^{4-6}$ The spectral domain accessible for ISB devices is limited on the short wavelength side by the available band offset in the heterostructure and the material transparency. To access the near-infrared spectral range, nitride heterostructures started to be intensively explored from the late 1990s. ${ }^{7}$ Thanks to their large conduction band offset, ISB absorption in the 1.3-4 $\mu \mathrm{m}$ range has been demonstrated in GaN/AIN QWs grown along the [0001] polar direction. ${ }^{8,9}$ Nitride heterostructures grown along the [0001] polar axis possess an intense internal electric field due to spontaneous and piezoelectric polarization. ${ }^{10}$ The built-in field can be extremely strong up to $10 \mathrm{MV} / \mathrm{cm}$ for $\mathrm{AlN} / \mathrm{GaN}$ QWs. ${ }^{11}$ The presence of this internal field complicates the design of ISB devices ${ }^{12}$ since it induces band bending effects and the formation of depletion/accumulation regions, while reducing the oscillator strength associated with the ISB transition. For optoelectronic applications, it is, therefore, desirable to reduce the internal electric field. This can be achieved by using III-nitride materials synthesized in the cubic

\footnotetext{
${ }^{\text {a)} E l e c t r o n i c ~ m a i l: ~ h o u s s a i n e . m a c h h a d a n i @ g m a i l . c o m ~}$
}

phase ${ }^{13-16}$ or by changing the growth direction to set the polarization vector at $90^{\circ} .{ }^{17}$

The later strategy has been implemented using nonpolar growth planes, namely, the $m$-plane $\{10 \overline{1} 0\}^{18}$ or the $a$-plane $\{11 \overline{2} 0\} .{ }^{19}$ However, growth of nonpolar III-nitrides is challenging due to the strong anisotropy of the surface properties, resulting in layers with a high density of crystalline defects. An alternative approach is the growth along semipolar planes, such as $\{11 \overline{2} 2\}$. The advantage of semipolar orientations is that they allow a considerable reduction of the internal electric field ${ }^{20}$ while presenting lower in-plane anisotropy with respect to non-polar surfaces. ${ }^{21}$ We have previously reported the observation of ISB absorption in non-intentionally doped semipolar GaN/AlN QWs using a photoinduced absorption spectroscopic technique relying on the photogeneration of electron-hole pairs in the QWs. ${ }^{22}$

In this work, we present a systematic study of the photoluminescence (PL) and ISB absorption of polar and semipolar GaN/AIN QWs grown by plasma-assisted molecular beam epitaxy (PAMBE). With respect to previous studies, efficient Si doping enabled the direct observation of the ISB absorption in both polar and semipolar samples with the same well thickness, grown during the same run. The optical properties are compared with structural characterizations and simulations, confirming a strong reduction of the internal field in the case of the semipolar orientation. By increasing the well thickness from 1.2 to $3 \mathrm{~nm}$, the peak ISB absorption wavelength is tuned from 1.5 to $3.3 \mu \mathrm{m}$ for semipolar samples, and from 1.5 to $2 \mu \mathrm{m}$ for polar samples. The spectral lineshape of the absorption is Gaussian in the case of the semipolar orientation, while it is a sum of Lorentzian functions for the polar orientation. 


\section{RESULTS AND DISCUSSION}

\section{A. Sample growth and structural characterization}

GaN/AlN semipolar QWs have been grown by PAMBE using the optimized growth conditions described in Ref. 22. Substrates consisted of 2 - $\mu$ m-thick (112)2)-oriented GaN layers deposited on $m$-sapphire by MOVPE. ${ }^{23}$ The PAMBE growth starts with a $100 \mathrm{~nm}$ thick GaN buffer layer. Then, a stack of $40 \mathrm{GaN} / \mathrm{AlN}$ QWs was deposited and overgrown with a 10-nm-thick AlN cap layer. The thickness of the AlN barriers and GaN QWs are summarized in Table I. To populate the ground electron state, the QWs were n-doped with $\mathrm{Si}$ at a nominal concentration $n_{Q W}=5 \times 10^{19} \mathrm{~cm}^{-3}$. For comparison purposes, polar samples with an identical structure were grown during the same runs on AlN on c-sapphire templates. It should be noted that these designs do not target the maximization of the ISB optical dipole, as it was done in Ref. 24 but they were chosen to evaluate the effect of the electric field in the semipolar GaN/AlN binary system.

The structural quality of the GaN/AlN semipolar MQWs has been studied by high resolution transmission electron microscopy (HRTEM).

Figure 1 displays the HRTEM images of semipolar GaN/AlN QWs viewed along the $[10 \overline{1} 0]$ and $[11 \overline{2} 3]$ zone axes. The QWs present a two-dimensional nature in both crystallographic orientations, and the interfaces are chemically sharp. The interface thickness fluctuations extend over $0.5-0.7 \mathrm{~nm}$. From larger images, we estimate a density of basal stacking faults of around $3 \times 10^{5} \mathrm{~cm}^{-1}$.

\section{B. Optical spectroscopic measurements}

\section{Photoluminescence spectroscopy}

PL spectra were collected at liquid-helium temperature using a $f=0.46 \mathrm{~m}$ Jobin Yvon HR spectrometer equipped with a liquid-nitrogen-cooled charge-coupled device (CCD) camera. The excitation was provided by a frequency-doubled continuous-wave $\mathrm{Ar}^{++}$laser at $\lambda=244 \mathrm{~nm}$.

Figure 2 shows the PL spectra of semipolar (top) and polar (bottom) samples measured at $4 \mathrm{~K}$. As expected, the PL peaks energy is red shifted when increasing the QW thickness in both polar and semipolar QWs. In polar QWs, the PL energy becomes smaller than the GaN gap energy for QW thickness larger than $1.8 \mathrm{~nm}$. This is due to the quantum confined Stark effect induced by the high internal electric field in the QWs. In semipolar QWs, the PL energy peak remains systematically above the GaN gap, attesting the reduction in

TABLE I. Structural parameters for the GaN/AIN polar and semipolar samples. $\mathrm{L}_{Q W}$ and $\mathrm{L}_{B}$ are the well and barrier thicknesses, respectively.

\begin{tabular}{lccc}
\hline \hline Sample & $\mathrm{L}_{Q W}(\mathrm{~nm})$ & $\mathrm{L}_{B}(\mathrm{Nm})$ & $\mathrm{n}_{Q W}\left(\mathrm{~cm}^{-3}\right)$ \\
\hline A & 1.2 & 5 & - \\
$\mathrm{B}$ & 1.8 & 3 & $5 \times 10^{19}$ \\
$\mathrm{C}$ & 2.1 & 3 & $5 \times 10^{19}$ \\
$\mathrm{D}$ & 2.25 & 3 & $5 \times 10^{19}$ \\
$\mathrm{E}$ & 2.6 & 3 & $5 \times 10^{19}$ \\
$\mathrm{~F}$ & 3 & 3 & $5 \times 10^{19}$ \\
\hline \hline
\end{tabular}
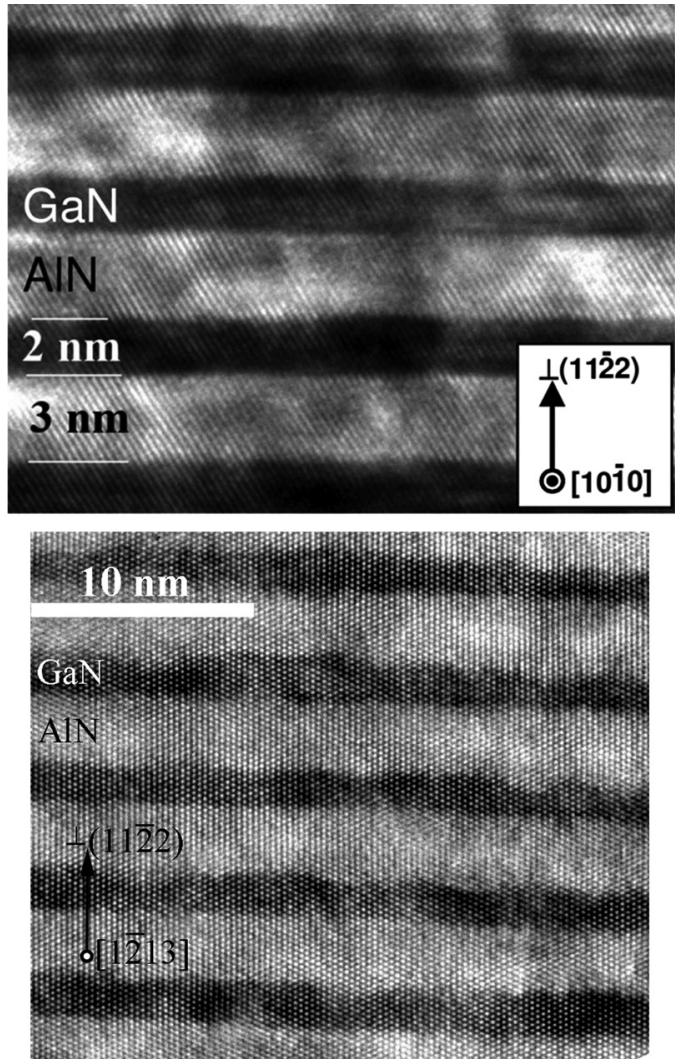

FIG. 1. Cross-section high-resolution TEM image of semipolar GaN/AIN quantum wells (sample C) viewed along the $[10 \overline{1} 0]$ and $[1 \overline{2} 13]$ zone axes.

the internal electric field in the QWs. The full width at half maximum (FWHM) of the PL spectra of semipolar QWs increases with the PL transition energy. This is due to $\mathrm{QW}$ thickness fluctuations, whose effect on linewidth increases when decreasing the well thickness. The value of the FWHM in semipolar QWs is 2 to 3 times larger than that in polar QWs as a result of the larger QW thickness fluctuations and to the presence of stacking faults.

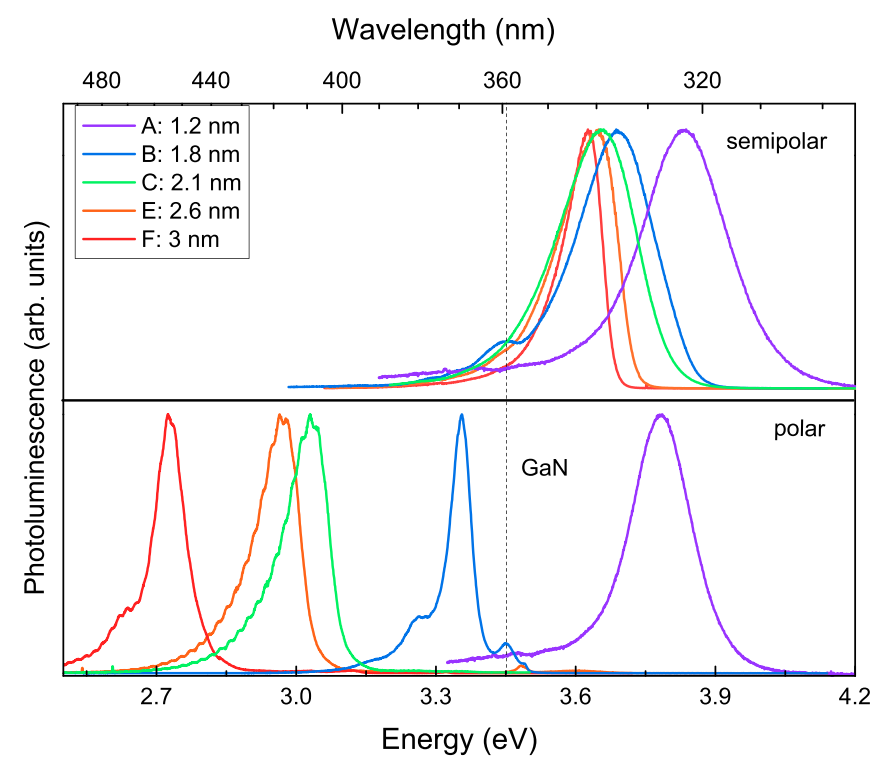

FIG. 2. Low-temperature (4K) photoluminescence of semipolar (top) and polar (bottom) GaN/AlN QWs. The dashed line indicates the GaN band gap. 


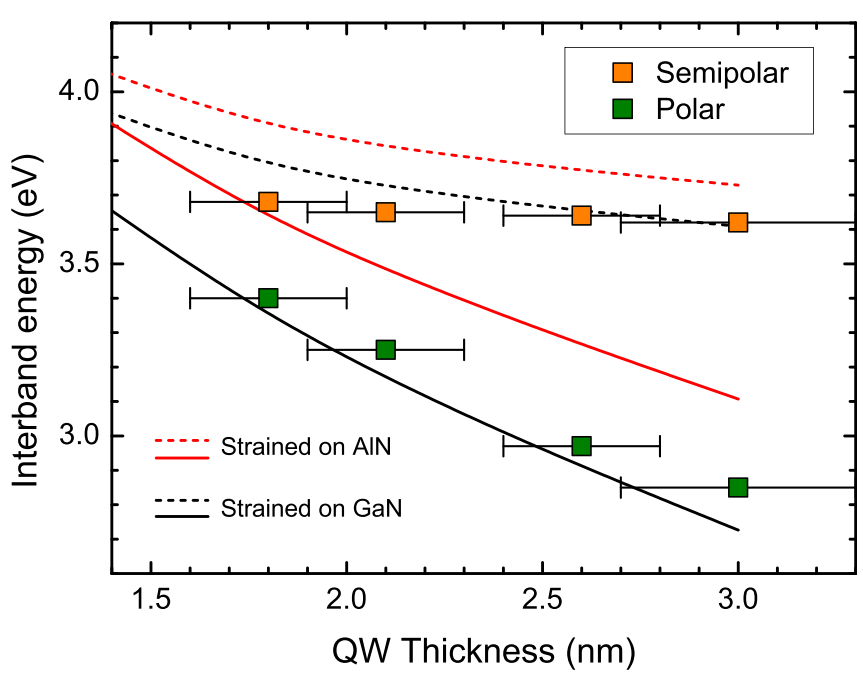

FIG. 3. Photoluminescence energy calculated as a function of QW thickness and strain state for polar and semipolar QWs.

PL results have been interpreted by comparison with calculations of electronic structure using the NEXTNANO ${ }^{3}$ 8-band-k.p Schrödinger-Poisson solver. The material parameters applied in the simulations are summarized in Ref. 25. As shown in Figure 3, the measured PL energies are in good agreement with calculations, with the experimental points from polar samples located within the band limited by the red and black solid lines, which correspond to the two extreme strain states (strained on GaN and strained on AlN). However, the emission wavelength from semipolar layers presents a certain red shift when compared with theoretical

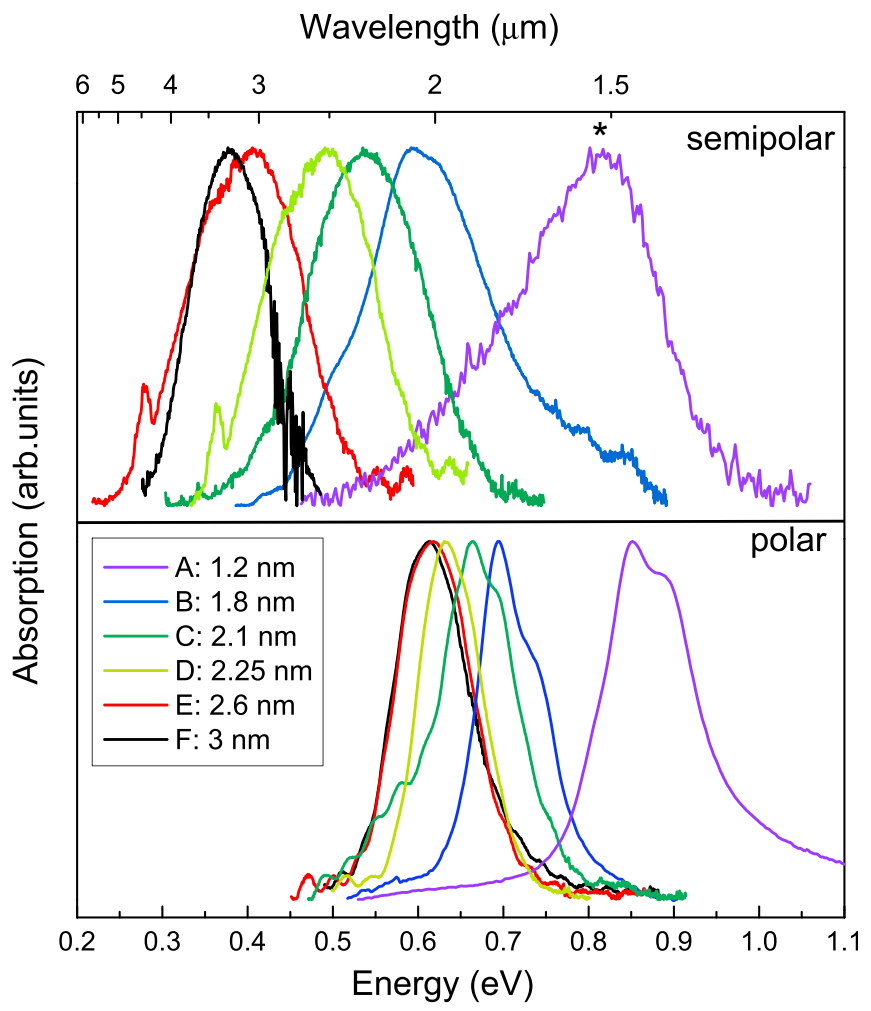

FIG. 4. Intersubband absorption spectra for semipolar (top) and polar (bottom) GaN/AlN QWs with different well thicknesses. The spectrum labeled with a star has been obtained by photoinduced absorption measurement.
TABLE II. ISB peak energy with corresponding broadening for polar and semipolar samples.

\begin{tabular}{lcccc}
\hline \hline Sample & & $\begin{array}{c}\text { QWs } \\
\text { thickness } \\
(\mathrm{nm})\end{array}$ & $\begin{array}{c}\text { ISB } \\
\text { energy } \\
(\mathrm{eV})\end{array}$ & $\begin{array}{c}\text { FWHM } \\
(\mathrm{meV})\end{array}$ \\
\hline A & $(0001)$ & 1.2 & 0.85 & 123 \\
& $(11 \overline{2} 2)$ & $\mathbf{1 . 2}$ & $\mathbf{0 . 8 1}$ & $\mathbf{1 9 5}$ \\
$\mathrm{B}$ & $(0001)$ & 1.8 & 0.69 & 100 \\
& $(11 \overline{2} 2)$ & $\mathbf{1 . 8}$ & $\mathbf{0 . 5 9}$ & $\mathbf{1 5 3}$ \\
$\mathrm{C}$ & $(0001)$ & 2.1 & 0.66 & 90 \\
& $(11 \overline{2} 2)$ & $\mathbf{2 . 1}$ & $\mathbf{0 . 5 4}$ & $\mathbf{1 5 6}$ \\
$\mathrm{D}$ & $(0001)$ & 2.25 & 0.63 & 87 \\
& $(11 \overline{2} 2)$ & $\mathbf{2 . 2 5}$ & $\mathbf{0 . 4 9}$ & $\mathbf{1 5 2}$ \\
$\mathrm{E}$ & $(0001)$ & 2.6 & 0.61 & 100 \\
& $(11 \overline{2} 2)$ & $\mathbf{2 . 6}$ & $\mathbf{0 . 4 0}$ & $\mathbf{1 6 0}$ \\
$\mathrm{F}$ & $(0001)$ & 3 & 0.61 & 105 \\
& $(11 \overline{2} 2)$ & $\mathbf{3}$ & $\mathbf{0 . 3 8}$ & $\mathbf{1 0 0}$ \\
\hline \hline
\end{tabular}

calculations, which can be attributed to carrier localization due to the presence of stacking faults and to larger thickness fluctuations than in the polar case.

\section{Intersubband spectroscopy}

The ISB absorption of the QWs was investigated using Fourier transform infrared spectroscopy (FTIR). The sample facets were polished at $45^{\circ}$ angle to form a multipass waveguide. The sample transmission for $p$ and $s$ polarized light was measured at room temperature. Figure 4 shows the ISB absorption spectra of polar and semipolar QWs. All samples show direct absorption of $p$-polarized light except the spectrum labeled with a star, which was measured by photoinduced absorption spectroscopy. For both polar and semipolar samples, the $\mathrm{e}_{1}-\mathrm{e}_{2}$ ISB absorption red shifts when increasing the QW thickness. It covers the 1.5-3.3 $\mu \mathrm{m}$ wavelength range for semipolar QWs, compared to $1.5-2 \mu \mathrm{m}$ for polar QWs. The ISB peak energy and the FWHM for all samples are indicated in Table II. As seen in Fig. 4, the ISB peak wavelength saturates at $2 \mu \mathrm{m}$ for polar QWs with a well thickness larger than $2.25 \mathrm{~nm}$, which is not the case for semipolar QWs.

Figure 5 compares the experimental $e_{1}-e_{2}$ ISB peak energy with simulations for semipolar and polar structures.

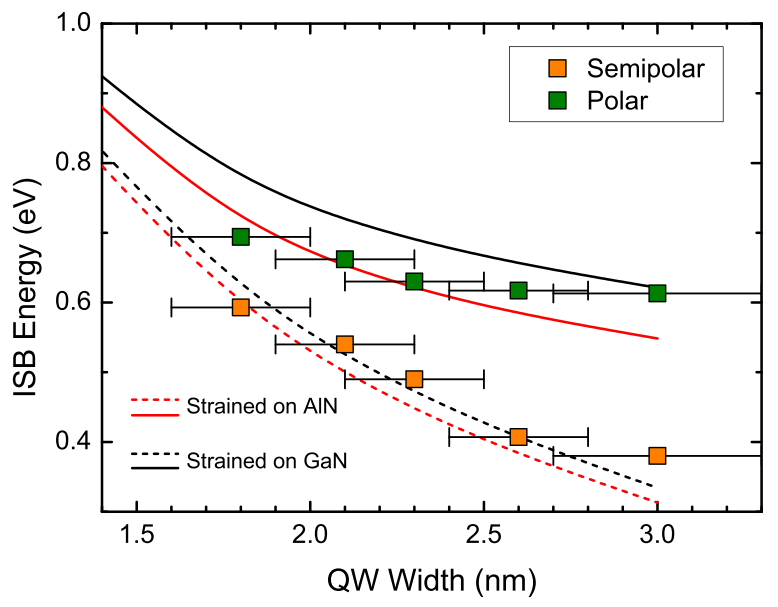

FIG. 5. Calculated and measured ISB absorption energy versus well thickness. 

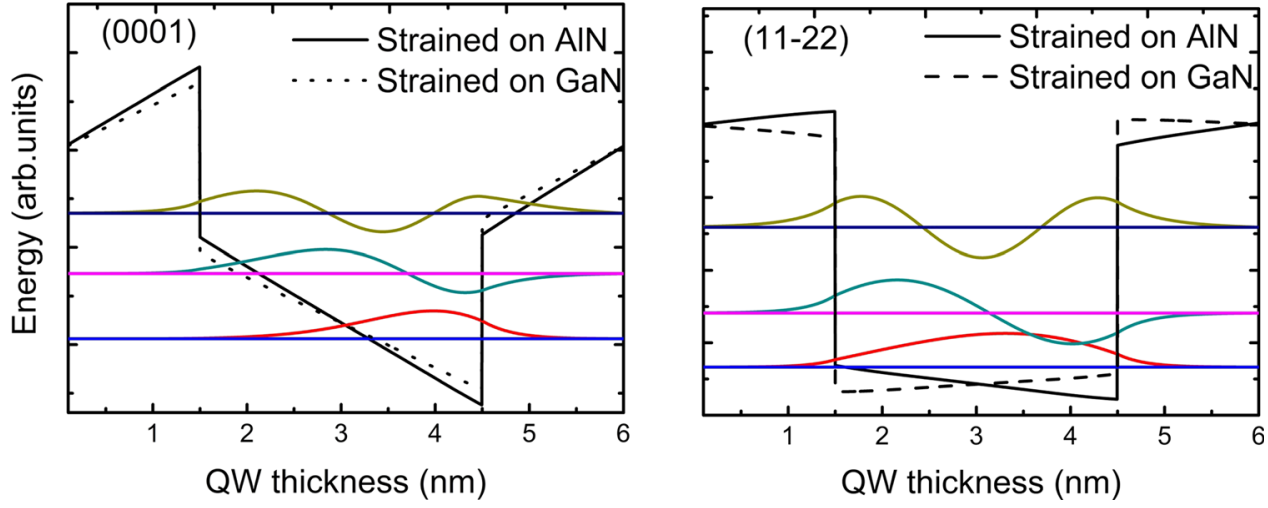

FIG. 6. Band diagram of (0001)- and $(11 \overline{2} 2)$-oriented GaN/AlN $(3 \mathrm{~nm} / 3 \mathrm{~nm})$ QWs in a superlattice. Solid lines correspond to simulations assuming the superlattice strained on AlN. The conduction band in the case of the wells being strained on $\mathrm{GaN}$ is included as a dashed line.
The simulation confirms a significant red shift of the transition energy in large semipolar QWs with respect to the polar case due to the reduction of the electric field in the wells. The saturation of the ISB transition for large QWs is explained by the fact that both $\mathrm{e}_{1}$ and $\mathrm{e}_{2}$ are located in the triangular section of the polar QWs, so that they are confined by the internal electric field in at distance smaller than the total QW thickness, as illustrated in Fig. 6. It should be noted that for the chosen barrier thickness $(3 \mathrm{~nm})$ there is no effect of the quantum coupling between adjacent QWs.

Assuming an infinite GaN/AlN superlattice, the internal fields in the QW and in the barrier are related to the respective polarizations and layer thicknesses by

$$
\begin{aligned}
F_{G a N} & =-\frac{\Delta P}{\varepsilon_{0}} \frac{L_{A l N}}{L_{A l N} \varepsilon_{G a N}+L_{G a N} \varepsilon_{A l N}}, \\
F_{A I N} & =\frac{\Delta P}{\varepsilon_{0}} \frac{L_{G a N}}{L_{A l N} \varepsilon_{G a N}+L_{G a N} \varepsilon_{A l N}},
\end{aligned}
$$

where $\Delta P$ is the difference between the total polarization (piezoelectric and spontaneous) of the well and the barrier, $\varepsilon_{A I N}$ and $\varepsilon_{G a N}$ are the dielectric constants for AIN and GaN, respectively, while $L_{A I N}$ and $L_{G a N}$ are the corresponding layer thicknesses. Taking $\varepsilon_{r}=\left(\varepsilon_{G a N}+\varepsilon_{A l N}\right) / 2$, for polar GaN/AIN MQWs we calculate $\Delta P / \varepsilon_{0} \varepsilon_{r}=10.4 \mathrm{MV} / \mathrm{cm}$ for MQWs strained on $\mathrm{AlN}$, and $\Delta P / \varepsilon_{0} \varepsilon_{r}=12.3 \mathrm{MV} / \mathrm{cm}$ for MQWs strained on GaN. For semipolar GaN/AlN MQWs, the value of $\Delta P / \varepsilon_{0} \varepsilon_{r}$ deduced from the simulations is 0.93 $\mathrm{MV} / \mathrm{cm}$ for MQWs strained on AIN and $-0.83 \mathrm{MV} / \mathrm{cm}$ for MQWs strained on GaN. The significant reduction in the polarization discontinuity is due to the fact that the spontaneous and piezoelectric polarization differences at the interfaces have opposite signs, the piezoelectric component being dominant in the MQWs strained on GaN resulting in negative $\Delta P$.

\section{Nature of ISB broadening}

By analyzing the ISB absorption spectra of polar and semipolar samples, we observe that the broadening and the spectral shape are very different. In semipolar QWs, the FWHM is around $100-195 \mathrm{meV}$, which is larger than that in polar QWs (90-105 meV). In addition, in polar samples with a QW thickness below $2.1 \mathrm{~nm}$ (samples A, B, and C), the ISB absorption spectra present well-defined multiple structures, which are not present in semipolar spectra. The multistructured lineshape of polar samples is well reproduced by a sum of Lorentzian curves. In contrast, for semipolar QWs, the ISB absorption resonance can be well fitted by a Gaussian function, as shown in Figure 7.

This difference is related to the sample structure and to the presence of internal electric field. In polar samples, the multistructured shape of the ISB absorption is interpreted as originating from absorption in well regions with different thickness, as discussed in Ref. 11. Due to internal electric field, for $\mathrm{QW}$ thickness below $\approx 2 \mathrm{~nm}$, a variation of the thickness of the QW of \pm 1 atomic layer translates into an ISB energy shift comparable to the broadening factor, and therefore results in structuring of the absorption spectrum instead of an inhomogeneous broadening.

For semipolar GaN/AlN QWs, the thickness fluctuations are larger ( $\pm 2-3$ atomic layers, as observed in Figure 1), but the energetic shift induced by each additional atomic layer is
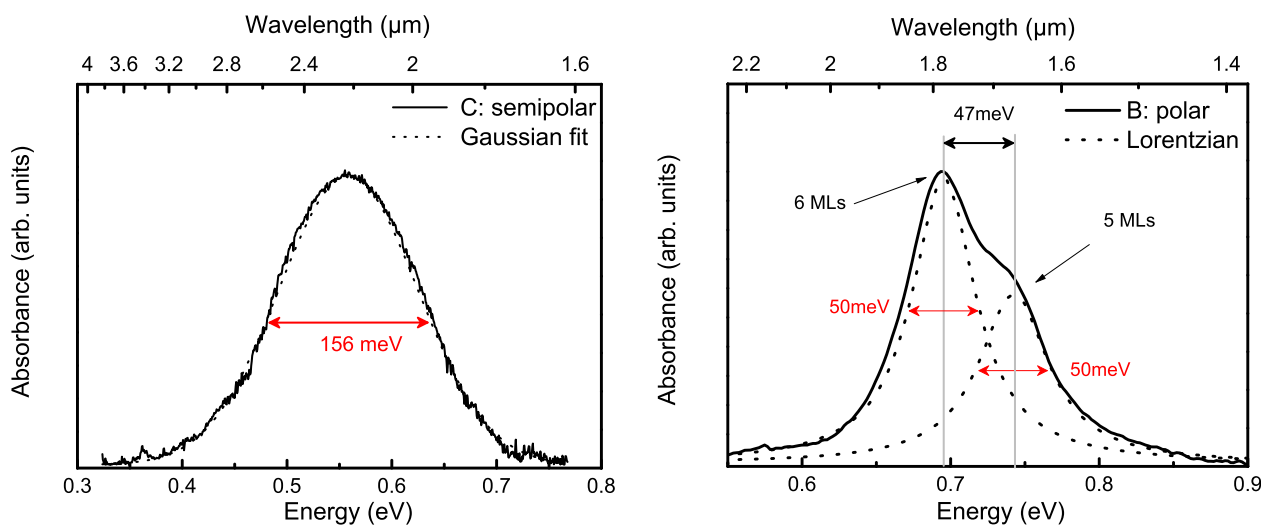

FIG. 7. (left) ISB absorbance of semipolar sample $\mathrm{C}$ and the corresponding Gaussian fit (dotted line). (right) ISB absorbance of polar sample B (full line), Lorentzian fitting curves (dotted lines). 
smaller, which results in an inhomogeneous broadening. Furthermore, the presence of stacking faults, as an additional perturbation of the bands, contributes to the absorption line broadening. We note that Gaussian lineshapes of the ISB absorption has also been observed in cubic $\mathrm{GaN} / \mathrm{Al}(\mathrm{Ga}) \mathrm{N}$ QWs ${ }^{16}$ and can be explained in the same manner.

\section{CONCLUSION}

In summary, we have systematically performed an experimental and theoretical study of ISB transitions in semipolar GaN/AlN quantum wells grown on (112 2$)$ GaN. The semipolar samples are compared with the polar samples grown in the same run. The ISB transition shows a significant red shift with respect to the polar case due to the reduction of the internal electric field in the quantum wells. The absorption peak is tuned from $1.5 \mu \mathrm{m}$ to $3.3 \mu \mathrm{m}$ by adjusting only the well width. The ISB absorption line shape exhibits a Gaussian shape which is explained by thickness fluctuations of the QWs, and by the presence of stacking faults.

\section{ACKNOWLEDGMENTS}

The author acknowledges the support by EC FETOPEN project Unitride under Grant Agreement \#233950 and by EU ERC-StG under project TeraGaN, Grant Agreement \#278428, and by the French National Research Agency under project COSNI, Grant No. ANR-08-BLAN-0298-01. The authors thank P. Lavenus for his assistance with photoluminescence measurements.

${ }^{1}$ L. C. West and S. J. Eglash, Appl. Phys. Lett. 46, 1156 (1985).

${ }^{2}$ J. Faist, F. Capasso, D. L. Sivco, C. Sirtori, A. L. Hutchinson, and A. Y. Cho, Science 264, 553 (1994).

${ }^{3}$ F. Capasso, C. Gmachl, D. L. Sivco, and A. Y. Cho, Phys. Today 55(5), 34 (2002).

${ }^{4}$ S. Gunapala and S. Bandara, Semicond. Semimetals 62, 197 (1999).
${ }^{5}$ B. F. Levine, S. D. Gunapala, J. M. Kuo, S. S. Pei, and S. Hui, Appl. Phys. Lett. 59, 1864 (1991).

${ }^{6}$ K. L. Tsai, K. H. Chang, C. P. Lee, K. F. Huang, J. S. Tsang, and H. R. Chen, Appl. Phys. Lett. 62, 3504 (1993).

${ }^{7}$ N. Iizuka, K. Kaneko, N. Suzuki, T. Asano, S. Noda, and O. Wada, Appl. Phys. Lett. 77, 648 (2000).

${ }^{8}$ K. Kishino, A. Kikuchi, H. Kanazawa, and T. Tachibana, Appl. Phys. Lett. 81, 1234 (2002).

${ }^{9}$ A. Helman, M. Tchernycheva, A. Lusson, E. Warde, F. H. Julien, K. Moumanis, G. Fishman, E. Monroy, B. Daudin, D. L. S. Dang, E. BelletAmalric, and D. Jalabert, Appl. Phys. Lett. 83, 5196 (2003).

${ }^{10}$ F. Bernardini and V. Fiorentini, Phys. Rev. B 57, R9427 (1998).

${ }^{11}$ M. Tchernycheva, L. Nevou, L. Doyennette, F. H. Julien, E. Warde, F. Guillot, E. Monroy, E. Bellet-Amalric, T. Remmele, and M. Albrecht, Phys. Rev. B 73, 125347 (2006).

${ }^{12}$ H. Machhadani, P. Kandaswamy, S. Sakr, A. Vardi, A. Wirtmüller, L. Nevou, F. Guillot, G. Pozzovivo, M. Tchernycheva, and A. Lupu, New J. Phys. 11, 125023 (2009).

${ }^{13}$ D. As, Microelectron. J. 40, 204 (2009).

${ }^{14}$ E. DeCuir, Jr., M. Manasreh, J. Schörmann, D. As, and K. Lischka, Appl. Phys. Lett. 91, 041911 (2007).

${ }^{15}$ E. DeCuir, Jr., M. Manasreh, E. Tschumak, J. Schörmann, D. As, and K. Lischka, Appl. Phys. Lett. 92, 201910 (2008).

${ }^{16}$ H. Machhadani, M. Tchernycheva, S. Sakr, L. Rigutti, R. Colombelli, E. Warde, C. Mietze, D. As, and F. Julien, Phys. Rev. B 83, 075313 (2011).

${ }^{17}$ J. Speck and S. Chichibu, MRS Bull. 34, 304 (2009).

${ }^{18} \mathrm{P}$. Waltereit, O. Brandt, A. Trampert, H. Grahn, J. Menniger, M. Ramsteiner, M. Reiche, and K. Ploog, Nature 406, 865 (2000).

${ }^{19}$ M. D. Craven, S. H. Lim, F. Wu, J. S. Speck, and S. P. DenBaars, Appl. Phys. Lett. 81, 1201 (2002).

${ }^{20}$ A. Romanov, T. Baker, S. Nakamura, and J. Speck, J. Appl. Phys. 100, 023522 (2006).

${ }^{21}$ L. Lahourcade, E. Bellet-Amalric, E. Monroy, M. Abouzaid, and P. Ruterana, Appl. Phys. Lett. 90, 131909 (2007).

${ }^{22}$ L. Lahourcade, P. K. Kandaswamy, J. Renard, P. Ruterana, H. Machhadani, M. Tchernycheva, F. H. Julien, B. Gayral, and E. Monroy, Appl. Phys. Lett. 93, 111906 (2008).

${ }^{23}$ P. D. Mierry, T. Guehne, M. Nemoz, S. Chenot, E. Beraudo, and G. Nataf, Jpn. J. Appl. Phys., Part 1 48, 031002 (2009).

${ }^{24}$ V. Jovanović, D. Indjin, Z. Ikonić, V. Milanović, and J. Radovanović, Solid state Commun. 121, 619 (2002).

${ }^{25}$ P. Kandaswamy, F. Guillot, E. Bellet-Amalric, E. Monroy, L. Nevou, M. Tchernycheva, A. Michon, F. Julien, E. Baumann, and F. Giorgetta, J. Appl. Phys. 104, 093501 (2008). 